\title{
First experimental prompt $\gamma$-ray spectra in fast neutron-induced fission of ${ }^{238} \mathrm{U}$
}

\author{
J.-M. Laborie ${ }^{1}$, G. Bélier ${ }^{1, \text { a }}$, J. Taieb ${ }^{1}$, A. Oberstedt ${ }^{2}$, and S. Oberstedt ${ }^{3}$ \\ 1 CEA, DAM, DIF, 91297 Arpajon, France \\ ${ }^{2}$ Horia Hulubei National Institute for Physics and Nuclear Engineering (IFIN-HH), 077125 Bucharest-Magurele, Romania \\ ${ }^{3}$ European Commission, Joint Research Center (IRMM), 2440 Geel, Belgium
}

\begin{abstract}
The knowledge of prompt fission $\gamma$-ray emission has been of major interest in reactor physics since few years, but it is also important in the understanding of the fission process. We present here measurements of prompt $\gamma$-ray spectra emitted in the fission of ${ }^{238} \mathrm{U}$. A simple experimental method was used to measure the prompt fission gamma-ray spectrum up to $10 \mathrm{MeV}$. In this approach, the gamma-rays are measured with a bismuth germanate (BGO) detector which offers two significant advantages with respect to other gamma-ray detectors: a high peak-to-total ratio and a high efficiency. The prompt fission neutrons are rejected by the time-of-flight technique between the BGO detector and a fission trigger given by a fission chamber. Prompt fission gamma-ray spectra were measured for $1.7,5.2$ and $15.6 \mathrm{MeV}$ incoming neutron energies at the CEA, DAM, DIF Van de Graaff accelerator.
\end{abstract}

\section{Introduction}

The knowledge of prompt fission $\gamma$-rays is important both for applications and for understanding the fission process. The $\gamma$-ray emission is the main source of the structures heating in nuclear reactors, and the prompt fission $\gamma$-rays is by far the less known. From a fundamental point of view, prompt $\gamma$-rays have to be measured in order to understand the angular momentum generation in the fission process [1].

But despite many experiments devoted to the study of fission, and especially fission fragment de-excitation, the emission of $\gamma$-rays in fission is still poorly known. Moreover most of the results were obtained for thermal neutron induced fission. For a very long time only results on the total $\gamma$-ray energy was obtained in the fission of ${ }^{235} \mathrm{U},{ }^{237} \mathrm{~Np}$ and ${ }^{232} \mathrm{Th}$ for incoming neutron energies between 1 and $15 \mathrm{MeV}$ [2], thanks to a $\mathrm{Gd}$ loaded neutron ball. It was measured relative to ${ }^{252} \mathrm{Cf}$ spontaneous fission total $\gamma$-ray energy, together with the neutron multiplicity. Nevertheless simulations performed on a similar detector [3], have shown that the contribution of prompt neutrons is amounts to be between 30 and $50 \%$, while typical corrections applied to subtract this contribution amounts to $15 \%$ [4]. Hence the absolute values of the total $\gamma$-ray energy has to be considered with care. Only very recently $[5,6] \gamma$-ray spectra were published for the fast neutron induced fission of ${ }^{235} \mathrm{U}$ and ${ }^{238} \mathrm{U}$. Nevertheless in the first experiment organic liquid scintillator were used, limiting the maximum energy of the emitted $\gamma$-rays to $4 \mathrm{MeV}$. In the second one it was still rather limited (to about $6 \mathrm{MeV}$ ) and was measured only for $1.7 \mathrm{MeV}$ impinging neutrons.
We report here measurements that were performed at the CEA/DIF $4 \mathrm{MV}$ accelerator at neutron energies of 1.7, 5.2 and $15.6 \mathrm{MeV}$.

\section{Experimental setup}

The main difficulty in measuring $\gamma$-ray spectra is related to the complicated response of the detectors to monoenergetic $\gamma$-rays, since several processes are involved. These processes are the photo-electric absorption, the Compton scattering and for energies greater than twice the electron mass the electron-positron pair-production. These last two processes lead or can lead to a partial energy deposition. Hence the measured spectra have to be unfolded in order to take into account this complicated response. Thus the choice of the detector is of prime importance because this unfolding procedure introduces uncertainties associated to the detector response simulation, and also leads to a propagation of statistical uncertainties mainly from the highest energies, to the lowest one. The parameter that has to be considered there is the peak-to-total ratio $\mathrm{P} / \mathrm{T}$. It is defined as the ratio of the full energy deposition peak area to the total spectrum area. The higher the P/T, the lower the spectrum distortion and the smaller the uncertainty associated to its correction.

Simulations of several kinds of detectors were performed with the GEANT 3.21 package, in order to quantify the $\mathrm{P} / \mathrm{T}$ ratio in the targeted energy domain $(1-10 \mathrm{MeV})$. These simulations were performed for different cylindrical detectors with same size (diameter and thickness of 2 inches). The results are plotted in Fig. 1 for $\mathrm{BGO}, \mathrm{BaF}_{2}, \mathrm{LaBr}_{3}, \mathrm{NaI}$ and $\mathrm{Ge}$ detectors. These simulations show that there is a gap between the BGO detector and all others. At $10 \mathrm{MeV}$ the $\mathrm{P} / \mathrm{T}$ ratio is about 5 times higher than for $\mathrm{LaBr}_{3}$ for example. Moreover the full-energy detection efficiency is also about 5 times higher

\footnotetext{
${ }^{a}$ e-mail: gilbert.belier@cea.fr
}

(C) The Authors, published by EDP Sciences. This is an Open Access article distributed under the terms of the Creative Commons Attribution License 4.0 (http://creativecommons.org/licenses/by/4.0/). 


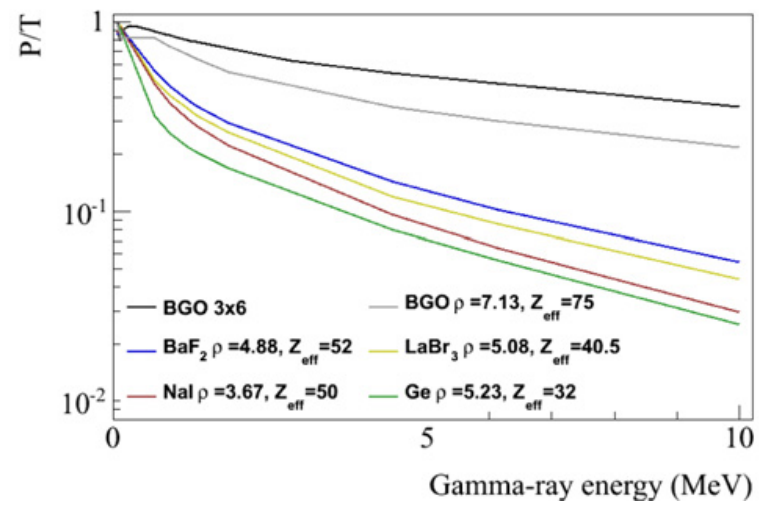

Figure 1. Simulations of the P/T ratio against $\gamma$-ray energy, for several detectors.

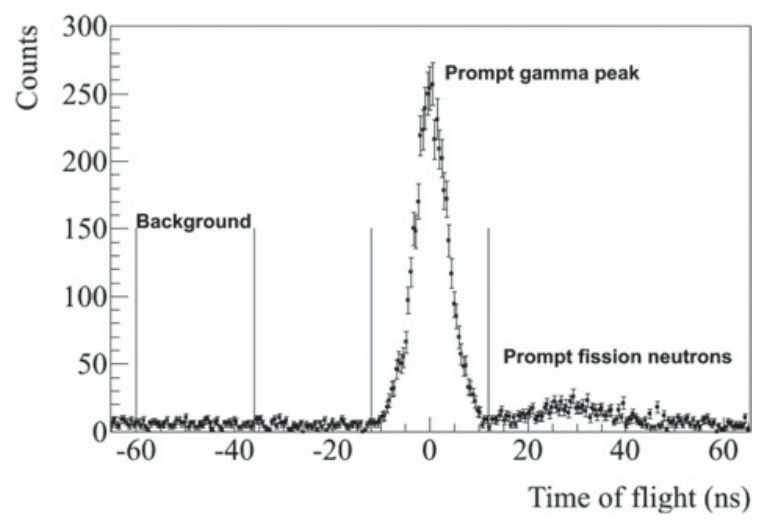

Figure 2. Time-Of-Flight spectrum obtained from the fission chamber and the BGO detector. The prompt gamma peak width allows to determine the FWHM time resolution.

than the $\mathrm{LaBr}_{3}$ efficiency. Hence, there is a cumulative effect on the final precisions, since the corrections are smaller and the statistical uncertainties also. Figure 1 also shows the results that were obtained for a BGO of bigger sizes ( 3 inches for the diameter, and 6 inches long), that was finally retained to perform the measurements. This choice was made despite the poor energy and time resolution of this detector. Nevertheless the main goal was to characterize the high energy part of the spectra, and to minimize the uncertainties at lower energies.

Usual neutron production reactions $(\mathrm{d}, \mathrm{T})$ and $(\mathrm{D}, \mathrm{T})$ were used in order to obtain neutrons of 1.7, 5.2 and $15.6 \mathrm{MeV}$ thanks to proton and deuteron beams. A multiplate fission chamber, containing a total ${ }^{238} \mathrm{U}$ mass of $14.4 \mathrm{~g}$, was used as an active target. This detector contains 220 samples made of $\mathrm{U}_{3} \mathrm{O}_{8}$ with an average thickness of $1.7 \mathrm{mg} / \mathrm{cm}^{2}$. The fission rate was typically $100 \mathrm{~s}^{-1}$. The $\mathrm{BGO}$ detector was placed at a distance ranging from 70 to $90 \mathrm{~cm}$, allowing to discriminate $\gamma$-rays from neutrons by the Time-Of-Flight (TOF) technique. It was shielded with $\mathrm{B}_{4} \mathrm{C}$ loaded polymer, in order to minimize capture events in the detector. Additional shielding was placed near the neutron production target in order to minimize the background $\gamma$-rays.

Figure 2 shows a typical TOF Spectrum, with a prompt $\gamma$-ray peak exhibiting a FWHM time resolution of $10 \mathrm{~ns}$. By gating the events by the window centred on this prompt peak, $99.5 \%$ of the fast fission neutrons were rejected. The TOF spectrum was also used to determine the $\gamma$-ray background contribution by gating the $\gamma$-ray events by an

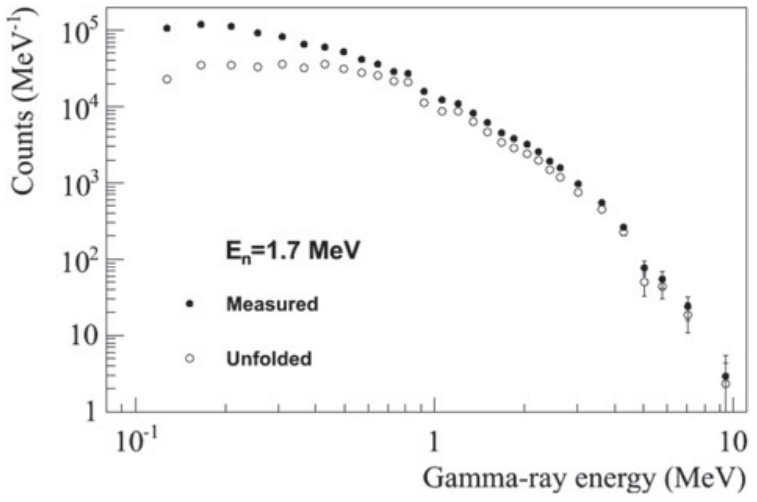

Figure 3. Measured (full circles) and unfolded (open circles) spectra for an incident neutron energy of $1.7 \mathrm{MeV}$.

appropriate time windows located just before the prompt fission $\gamma$-ray peak. The contribution of delayed $\gamma$-rays was estimated to be less than $1 \%$ in the final prompt $\gamma$-ray spectrum.

\section{Unfolding procedure and results}

The resulting measured spectrum obtained for neutron energies of $1.7 \mathrm{MeV}$ is shown in Fig. 3 (full circles). In order to unfold the spectrum for partial energy deposit, simulations of the whole setup including fission chamber, air, shieldings) were performed with the PENELOPE code [7].

A very simple procedure was then applied to unfold the measured spectrum. Starting from the highest measured energy, the energy bin content is used to compute the contribution of simulated partial energy deposit spectrum associated to this energy, which is then subtracted to the lower energy bins. The next energy bin is thus corrected and the new bin content is used again to correct the lower energies, and so on down to the lowest measured energy. At each step statistical uncertainties were propagated. Figure 3 shows the resulting unfolded spectrum obtained at neutron energy of $1.7 \mathrm{MeV}$. One can see that the both spectra are very close to each other at the highest energies. This justifies the procedure starting from an uncorrected bin, since the corrections applied at high energies are smaller than the statistical uncertainties. Moreover one can see that the statistical uncertainties remain very low at low energy. This is due to the high $\mathrm{P} / \mathrm{T}$ ratio which prevents high energies statistical uncertainties to contribute significantly despite lower statistics.

This unfolded spectrum can then be used to obtain the emission spectrum, by using the simulated/measured full energy peak detection efficiency. Figure 4 shows the obtained emission spectra obtained at incident neutron energies of 1.7 and $5.2 \mathrm{MeV}$. No differences are observed for these 2 energies. They are also compared to the results of V.V. Verbinski [8] obtained in the thermal neutron induced fission of ${ }^{235} \mathrm{U}$. Except for the lowest energies (below $400 \mathrm{keV}$ ) this last spectrum is again very similar to the spectrum obtained for the fission of ${ }^{238} \mathrm{U}$.

In Fig. 5 the emission spectrum obtained in this work at neutron energies of 1.7 and $15.6 \mathrm{MeV}$ are compared. This time one can observe a significant deviation beyond $\gamma$-ray energies of $2 \mathrm{MeV}$, while the 2 spectra looks very 


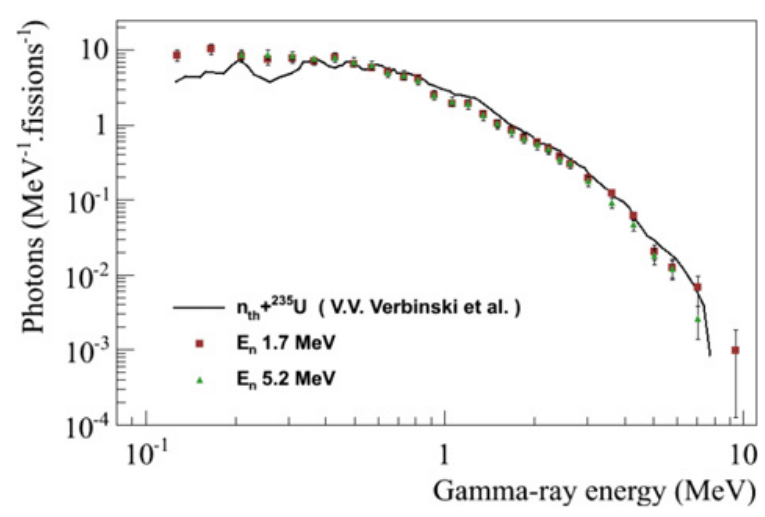

Figure 4. Emission spectra in the fission of ${ }^{238} \mathrm{U}$ obtained at neutron energies of $1.7 \mathrm{MeV}$ (brown squares) and $5.2 \mathrm{MeV}$ (green triangles). For comparison the prompt fission $\gamma$-ray spectrum obtained by V.V. Verbinski in the thermal neutron induced fission of ${ }^{235} \mathrm{U}$ is shown (black line).

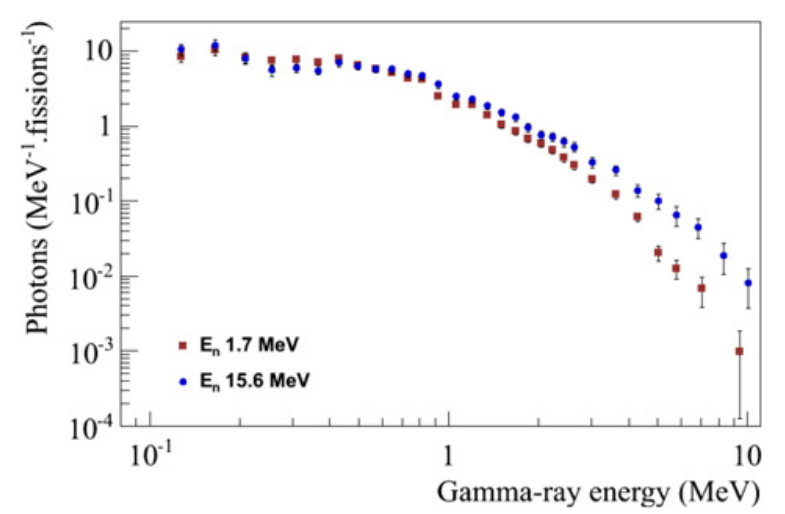

Figure 5. Emission spectra in the fission of ${ }^{238} \mathrm{U}$ obtained at neutron energies of $1.7 \mathrm{MeV}$ (brown squares) and 5.2 MeV (blue circles).

similar at the lowest energies, the spectrum corresponding to $15.6 \mathrm{MeV}$ is harder than the one measured at $1.7 \mathrm{MeV}$.

\section{Conclusion}

For the first time a change of the spectrum of emitted prompt fission $\gamma$-rays against neutron energy has been observed. This was possible by using a high $\mathrm{P} / \mathrm{T}$ and high efficiency BGO detector. This choice allows to characterize the spectra up to $10 \mathrm{MeV}$ with reasonable statistical uncertainties, and minimizes uncertainties propagation to lower energies. The spectrum could be measured for 3 different energies, 1.7, 5.2 and 15.6 MeV. The average energies together with the average multiplicities were extracted from these spectra, and the results are summarized in Table 1. The first two spectra do not differ while the third one has a higher average energy due to a stronger high energy component.

More measurement should be performed done to conclude on the behaviour of prompt $\gamma$-ray emission against neutron energy, and unfortunately there is no experiment in the neutron energy range explored here with sufficient precision above $2 \mathrm{MeV} \gamma$-ray energies.

The only available data are the total prompt $\gamma$-ray energy measured in the fission of ${ }^{235} \mathrm{U},{ }^{237} \mathrm{~Np}$ and ${ }^{232} \mathrm{Th}$ by J. Fréhaut [2], relative to the total $\gamma$-ray energy in the spontaneous fission of ${ }^{252} \mathrm{Cf}$. Figure 6 (top view) shows the
Table 1. Mean values of multiplicity and photon energy of prompt fission $\gamma$-rays in the fission of ${ }^{238} \mathrm{U}$ induced by $1.7,5.2$ and $15.6 \mathrm{MeV}$ neutrons.

\begin{tabular}{|c|c|c|}
\hline $\begin{array}{c}\text { Neutron energy } \\
(\mathrm{MeV})\end{array}$ & $\begin{array}{c}\text { Average } \\
\text { multiplicity }\end{array}$ & $\begin{array}{c}\text { Average energy } \\
(\mathrm{MeV})\end{array}$ \\
\hline 1.7 & $6.32(18)$ & $0.91(3)$ \\
\hline 5.2 & $6.50(18)$ & $0.86(3)$ \\
\hline 15.6 & $7.26(20)$ & $1.24(5)$ \\
\hline
\end{tabular}
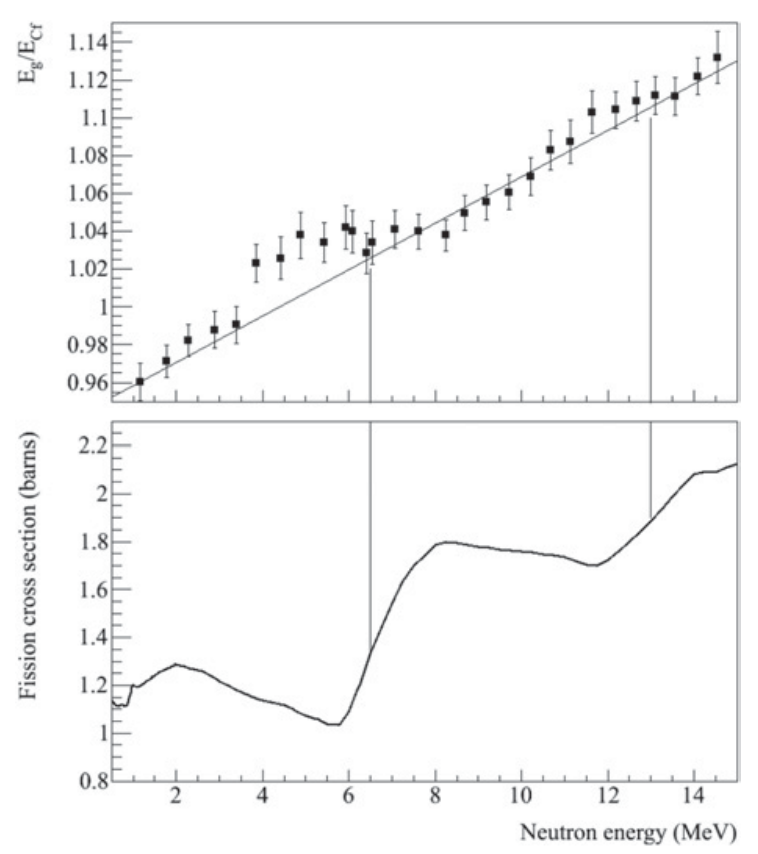

Figure 6. Top: total prompt $\gamma$-ray energy measured in the fission of ${ }^{235} \mathrm{U}$ against neutron energy. Bottom: ${ }^{235} \mathrm{U}$ fission cross section. Both figures are from Ref. [2].

result of the measurement performed on ${ }^{235} \mathrm{U}$ for neutron energies comprised between 1.1 and $14.5 \mathrm{MeV}$. On the bottom of Fig. 6 the fission cross section is plotted. Vertical lines are plotted in order to locate the opening of the second and third chance fission channels. On the top view a straight line has been traced in order to show the general trend of the total $\gamma$-ray energy. One can see that there is a systematic deviation of the total $\gamma$-ray energy from this general trend in the neutron energy domains preceding the second and third chance fission. This behaviour could be understood by assuming that the compound nucleus emits $\gamma$-rays before the fission since it can not emit an extra neutron. As soon as an extra neutron can be emitted (second and third chance fission) the total $\gamma$-ray energy goes back to the general trend, due to the emission by fission fragments. Our data do not allow to confirm this hypothesis since we have only 3 different neutron energy. Moreover it should be shown that the rise of the total $\gamma$-ray energy is associated to a rise of the average energy, and not to an augmentation of the $\gamma$-ray multiplicity only. In our measurement we observe both effects since at $15.6 \mathrm{MeV}$ the average multiplicity is about 1 unit greater than at 1.7 and $5.2 \mathrm{MeV}$. Hence a systematic characterization of the prompt fission $\gamma$-ray spectrum has to be performed in order to understand the rise of the average $\gamma$-ray energy per photon and of the total $\gamma$-ray energy. The rise of the average energy can not be explained by an angular momentum effect because it would lead to more 
E2 transitions along the yrast lines. Such transitions have relatively low energies, and correspond to the emission of discrete lines. For now this is the only assumption that has been done to explain neutron- $\gamma$ emission competition in fission, but it can not explain the high energy component which is observed in our measurement.

\section{References}

[1] H. Nifenecker et al., Nucl. Phys. A 189, 285-304 (1972)

[2] J. Fréhaut, A. Bertin and R. Bois, Proceedings of the Conference on Nuclear Data for Science and Technology (1983), 78-81
[3] S. Vayre PhD 03/27/2015 Univ. Paris-Sud, France

[4] H. Nifenecker et al. Nucl. Phys. A 189, 285 (1972)

[5] E. Kwan et al. Nucl. Instr. Methods A 688, 55 (2012)

[6] M. Lebois et al. Phys. Rev. C 92, 034618 (2015)

[7] F. Salvat, J.M. Fernandez-Varea, and J.Sempau, PENELOPE-2011, A Code System for Monte Carlo Simulation of Electron and Photon Transport, http: //www . oecd-nea.org/tools/abstract/ detail/NEA-1525. For the 2008 version, see https : //www . oecd-nea.org/science/pubs/ 2009/nea6416-penelope.pdf

[8] V.V. Verbinski, H. Weber, R.E. Sund, Phys. Rev. C7, 1173 (1973) 